\title{
Excitation of vortex-antivortex pairs in thin superconducting films and superlattices
}

\author{
P. Mikheenko \\ Department of Physics, University of Oslo, P.O.Box 1048, Blindern, 0316, Oslo, Norway
}

Direct imaging of the accumulation of magnetic flux and antiflux resulted from the excitation of vortex-antivortex pairs inside thin superconducting films is reported. Thin-film superconductors, like $\mathrm{YBa}_{2} \mathrm{Cu}_{3} \mathrm{O}_{7} / \mathrm{PrBa}_{2} \mathrm{Cu}_{3} \mathrm{O}_{7}$ superlattices or $\mathrm{NbN}$ films grown by pulsed laser deposition were used in experiments. The superlattices provide enhanced pinning for vortices, facilitating imaging of accumulated flux, and feature nanoscale fractures ideal for excitation of vortex-antivortex pairs. The idea of experiment is to record images using specific magneto-optical mode that allows distinguishing between positive and negative magnetic field in the sample. Two types of flux-antiflux patterns are observed. In one type, flux and antiflux are entering on permanent defects, like nano-fractures formed in the process of film deposition. In another type, flux and antiflux patterns are formed by application of strong localized magnetic field and not are linked to permanent defects. In first type, the amount of flux and antiflux entering superconductor from defect is strongly affected by external magnetic field. In the second type, the permanent frozen pattern is hardly influenced by the field. An unusual and dramatic effect of the excitation of flux-antiflux dendritic avalanches is also reported.

\section{INTRODUCTION}

Superconducting thin films have wide range of applications in electronics. They are used, for example, in thin-film SQUD-on-tip microscope [1], and thin films of topological superconductors can solve existing problems in quantum computing [2]. Based on thin-film technology, pulse laser-deposited superconducting tapes of second generation are already in trial use in cable and magnets applications [3]. Advanced preparation of thin films is based on nano-techniques designed to improve their critical current density, especially in strong magnetic fields [4]. Still, physics of magnetic behaviour of superconducting films is not ful- 
ly understood and continue to surprise by unusual effects. One of these effects is generation of vortex-antivortex pairs inside superconducting films.

One can expect that applying magnetic field of certain direction perpendicular to superconducting film would introduce magnetic flux of the same direction entering from a sample edge. Such entrance is well described theoretically for most of important sample's geometries, starting with simplest disc geometry [5], and confirmed in multiple experiments. However, presence of defects or areas of suppressed superconductivity would lead to somewhat unexpected effect of the excitation of magnetic field loops with equal amount of quantized flux and antiflux in the plane of the sample. The current flowing in the film would increase size of these loops moving vortices and antivortices in opposite direction. If pinning is strong, vortices will be trapped on pinning centers creating localized areas with equal, and opposite in direction, amount of magnetic flux. If pinning is weak and the dissipation of the energy during the flux motion is high, it could lead to thermomagnetic instability.

The mechanism of excitation of vortex-antivortex pairs was described as early as in 1983 in [6] explaining formation of localized resistive domains on artificial areas of suppressed superconductivity inside the film. A modern demonstration of this effect with low-temperature scanning tunneling microscope can be found in [7]. This technique, however, is not ideal for samples with large in-plane dimensions. Another technique, namely magneto-optical imaging (MOI) [8-10] is more suitable for this. MOI was already used to image accumulation of large amount of flux and antiflux on artificially produced slits [11]. The application of this technique to samples with naturally formed nano-fractures and point-like defects is described in the following sections.

\section{EXPERIMENTAL}

The main sample used in experiments was a $\mathrm{YBa}_{2} \mathrm{Cu}_{3} \mathrm{O}_{7} / \mathrm{PrBa}_{2} \mathrm{Cu}_{3} \mathrm{O}_{7}$ ( $\mathrm{YBCO} / \mathrm{PrBCO}$ ) superlattice prepared by pulsed laser deposition on a $\mathrm{SrTiO}_{3}$ substrate. In this superlattice, 48 layers of $\mathrm{YBa}_{2} \mathrm{Cu}_{3} \mathrm{O}_{7}$ (YBCO) of the thickness of about $60 \mathrm{~nm}$ were separated by incomplete, less than one-unit-cell thick, layers of $\mathrm{PrBa}_{2} \mathrm{Cu}_{3} \mathrm{O}_{7}$ ( $\mathrm{PrBCO}$ ) used to nucleate defects for increasing pinning in $\mathrm{YBCO}$ $[4,12]$. The details of the film growth and its behavior in magnetic field are described in [13]. The specific feature of the film is formation of nano-fractures leading to channeled magnetic flux flow [13].

In MOI technique, a Bi-substituted iron garnet indicator film was placed on top of the film, and together they were attached to cold finger of an optical cryostat. Measurements of $\mathrm{YBCO} / \mathrm{PrBCO}$ superlattice were performed using liquid nitro- 
gen or liquid helium as coolant. Another sample was a $\mathrm{NbN}$ film with critical temperature of about $16 \mathrm{~K}$. MOI images were recorded using a field-cooled procedure or slightly uncrossed polarizer and analyzer to distinguish between positive and negative magnetic fields.

\section{RESULTS AND DISCUSSION}

An example of magnetic flux entering $\mathrm{YBCO} / \mathrm{PrBCO}$ superlattice on internal defects as described above, is shown in Fig. 1. There are several nano-fractures in the superlattice. When external field is increased, superconductor is filled with positive (white) and negative (dark) magnetic field. It is important to note that fractures themselves are so narrow (less than one micron) that they are not resolved in imaging. What is seen is magnetic flux in superconductor close to the fractures. With generation of vortex-antivortex pairs, amount of positive magnetic flux trapped in superconductor is equal to amount of negative flux. This is qualitatively demonstrated by Fig. 2, which contains three-dimensional images of the areas selected by red frames in Fig. 1. In these plots, the intensity of the light is plotted along the z-axis.

Magneto-optical image in Fig. 1 was recorded at polarizer and analyzer uncrossed by 5 degrees, which is suitable to distinguish between positive (white) and negative (dark) magnetic fields. The temperature of the record was $3.7 \mathrm{~K}$, and the applied magnetic field was $4.3 \mathrm{mT}$.

The areas of positive and negative flux in the figure have specific dumb-bell shapes directed along a line. However, in some cases, positive and negative parts are at 90 degrees, as it is marked by red arrow in the figure, or just one part could be present if another is erased by a closely-spaced singularity as in a feature to the bottom-left from the upper red frame. To the right of the same frame, another configuration is seen of the central dark area surrounded by the two bright islands.

Such wealth of configurations reflects length and positions of nano-fractures in the sample and also the flow of supercurrent circulating in the film in presence of magnetic field. If current flows perpendicular to a nano-fracture, it starts bending around it and drags excited vortices and antivortices in different parts, first along the fracture, and further perpendicular to it into superconductor, where they become trapped on pinning centers.

If two perpendicular fractures are connected in a point, the distribution of flux will be at the right angle, like it is pointed to by the red arrow in Fig. 1. For an isolated linear nano-fracture with perpendicular current flow, the largest current density is close to the edges, and the lowest is in the middle. Therefore, no separated 
flux in superconductor is seen in the middle, and its amount gradually increases towards the edges. The distribution of flux around nano-fractures is strongly affected by applied field. At higher than in Fig. 1 field, patterns of trapped flux start merging with corresponding annihilation of flux and antiflux, and they are overlapped by magnetic flux advancing from the edges.

Completely different pattern could appear when superconducting film is exposed to localized magnetic field, strong enough to suppress the superconductivity. One of the examples of such a pattern is shown in Fig. 3. It is a stripy line with periodical repetition of the fragments of positive and negative magnetic flux, seen on a background of weak horizontal and vertical lines. These lines also appear along a network of nano-fractures. The sample in this figure is another $\mathrm{YBCO} / \mathrm{PrBCO}$ superlattice with four, much thicker than those in the superlattice in Figs. 1,2, layers of YBCO of $750 \mathrm{~nm}$.

The stripy line in Fig. 3 reminds a track left in superconductor by a particle. In fact, it was first treated like the track of high-energy particle. If such particle enters superlattice, it starts destroying superconductivity inside the film. In a very short time, superconductivity recovers trapping positive and negative magnetic flux formed during suppression of superconductivity. This process periodically repeats along the track while particle moves inside the film, resulting in a stripy pattern that can be seen by magneto-optical imaging. Period of this pattern is defined by the speed of the particle and by relaxation time of superconductor. Knowing the volume of the film with suppressed superconductivity allows calculating amount of energy deposited into the material. The calculation gives realistic agreement with recovery time of superconductor (about $2 \mathrm{~ns}$ ) and reveals a very high amount of energy deposited into superconductor, of about $160 \mathrm{GeV}$, which, judging by track appearance, is likely to correspond to a dark-matter particle. The idea was abandoned when it was found that the position of the track exactly coincides with the position of the edge of MOI indicator film that previously has been positioned there before being shifted to a different place during the orientation of sample.

Similar to the accumulations of positive and negative flux in Figs. 1,2, there is a balance between flux and antiflux along the track in Fig. 3, but the flux here behaves differently from the patterns in the first two figures, in that it virtually insensitive to external magnetic field. For example, Fig. 4 shows the same part of the sample as in Fig. 3, but in a higher magnetic field of $6.8 \mathrm{mT}$. The background is completely changed, while stripy pattern remains seemingly the same in the places, where it is not erased by magnetic flux advancing from the nano-fractures.

Such insensitivity to magnetic field could be explained by the fact that after removal of the edge of MOI film, superconductivity fully recovers below the stripy pattern and keeps the frozen flux, whose density is too low to significantly 
affect the local critical current density. In nano-fractures, however, local critical current density is always zero.

At high field, or even at zero field after the application of high field, the excitation of vortex - antivortex pairs seems to have little influence on global distribution of magnetic flux or energy stored in the superconductor. An example of one of the distributions of trapped magnetic flux in main superlattice composed of 48 layers of YBCO at liquid nitrogen temperature of $77.3 \mathrm{~K}$ is shown in Fig. 5. The places of generation of vortices and antivortices provide just a weak modulation of the density of a large amount of flux trapped in the film.

Still, in some cases, centers of the generation of vortices and antivortices can play important role in the dynamics of flux flow in superconductors. This possibility is linked to formation of thermomagnetic instabilities [8-10]. In fact, mechanism of the excitation of vortices and antivortices was suggested following observation of thermomagnetic instabilities leading to formation of resistive domains in superconducting films with large transport current [6]. In that case, flux and antiflux formed on artificial point-like defects inside the film were accelerating in opposite directions due to the Lorentz force from the transport current.

Thermomagnetic instabilities are also frequent in thin films in external magnetic field [8-10], but in this case, one could expect instabilities leading to abrupt penetration of magnetic flux of the same direction as applied magnetic field. Typically, it happens like this, but not always. Fig. 6 shows unusual cases when pairs of dendritic flux avalanches with magnetic flux of opposite directions are coming from the same points in the sample (marked by blue arrows), which are the points of the excitation of vortex - antivortex pairs. The dendrites are of different color (dark and white), and the imaging was, again, done with slightly uncrossed polarizer and analyzer to distinguish between positive and negative magnetic field.

The images in Fig. 6 are related to a study of ray-optics effects in magnetic flux flow in superconductors [14]. The fragments of normal stripes affecting flux flow are seen in the upper parts of the plots. The thermomagnetic avalanches coming from the centers of the excitation of vortex - antivortex pairs may disturb flux flow under the investigation, and could be harmful for any electronic devices that may be patterned in vicinity of these centers.

\section{SUMMARY}

Direct imaging of positive and negative trapped magnetic flux, as well as dendritic avalanches associated with excitation of vortex-antivortex pairs on internal 
defects in superconducting films is reported. The local suppression of superconductivity by strong magnetic field leading to stripy features of trapped magnetic flux with balanced amount of flux and antiflux is imaged. The difference in sensitivity of different flux patterns formed by excitation of vortex - antivortex pairs to external magnetic field is revealed. The importance of magneto-optical study allowing monitoring local distribution of magnetic flux is superconductors is emphasized.

\section{REFERENCES}

[1] Finkler A, Segev Y, Myasoedov Y, Rappaport ML, Ne'eman L, Vasyukov D, Zeldov E, Huber ME, Martin J, Yacoby A (2010) Self-aligned nanoscale SQUID on a tip. Nano Lett 10(3): 1046-1049

[2] Zhang P, Yaji K, Hashimoto T, Ota Y, Kondo T, Okazaki K, Wang Z, Wen J, Gu GD, Ding H, Shin S1 (2018) Observation of topological superconductivity on the surface of an ironbased superconductor. Science 360(6385):182-186. https://doi.org/10.1126/science.aan4596

[3] Samoilenkov S, Molodyk A, Lee S, Petrykin V, Kalitka V, Martynova I, Makarevich A., Markelov A, Moyzykh M, Blednov A (2015) Customised 2G HTS wire for applications. Supercond Sci Technol 29(2):024001

[4] Crisan A, Dang VS, Mikheenko P (2017) Nano-engineered pinning centres in YBCO superconducting films. Physica. C 553:118-132

[5] Mikheenko PN, Kuzovlev YuE (1993) Inductance measurements of HTSC films with high critical currents. Physica C 204(3-4):229-236

[6] Ivanchenko YuM, Mikheenko PN (1983) New mechanism of penetration of vortices into current-saturated superconducting films. Sov Phys JETP 58(6):1228-1234

[7] Ge JY, Gladilin VN, Tempere J, Devreese J, Moshchalkov VV (2017) Controlled Generation of Quantized Vortex-Antivortex Pairs in a Superconducting Condensate. Nano Lett 17(8): 5003-5007

[8] Johansen TH, Shantsev DV (eds) (2004) Magneto-Optical Imaging. Kluwer Academic Publishers, Dordrecht

[9] Jooss Ch, Albrecht J, Kuhn H, Leonhardt S, Kronmüller H (2002) Magneto-optical studies of current distributions in high-Tc superconductors. Rep Prog Phys 65(5):651-788

[10] Mikheenko P, Yurchenko VV, Cardwell DA, Shi YH, Johansen TH (2013) MagnetoOptical Imaging of Superconductors for Liquid Hydrogen Applications. Journal of superconductivity and novel magnetism 26(5):1499-1502

[11] Baziljevich M, Johansen TH, Bratsberg H, Shen Y, Vase P (1996) Magneto-optic observation of anomalous Meissner current flow in superconducting thin films with slits. Appl Phys Lett 69(23):3590-3592

[12] Crisan A, Dang VS, Mikheenko P, Tse YY, Sarkar A, Bowen J, Abell JS (2009) Pinning potential in thick $\mathrm{PrBa}_{2} \mathrm{Cu}_{3} \mathrm{O}_{\mathrm{y}} / \mathrm{YBa}_{2} \mathrm{Cu}_{3} \mathrm{O}_{\mathrm{x}}$ quasi-multilayers. Physica. C 470(1):55-60

[13] Mollatt HJS, Qureishy TH, Crisan A, Dang VS, Mikheenko P (2018) Flux channeling in $\mathrm{YBa}_{2} \mathrm{Cu}_{3} \mathrm{O}_{7}$ superlattices. In: Proceedings of International conference Nanomaterials: Applications and Properties NAP 9-14 September 2018, Sumy State University 3:03TFNMC09

[14] Mikheenko P, Johansen TH, Chaudhuri S, Maasilta IJ, Galperin YM (2015) Ray optics in flux avalanche propagation in superconducting films. Phys Rev B 91(6):060507(R) 


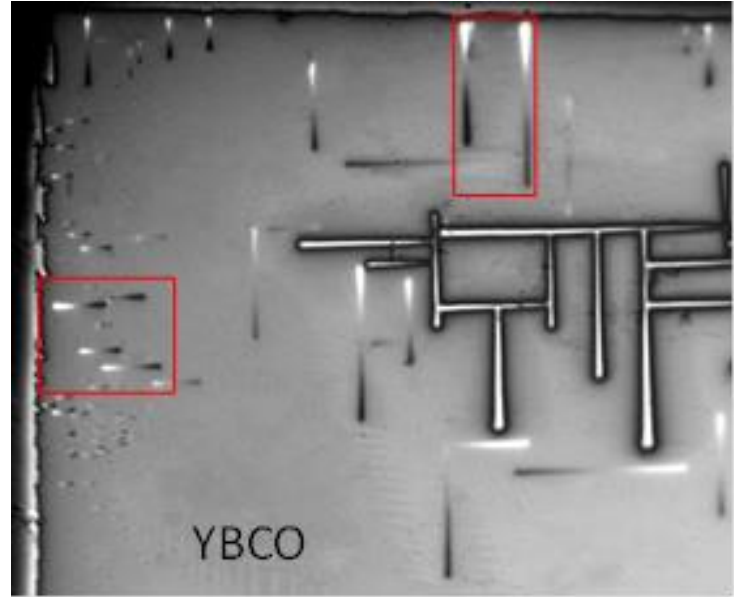

Figure 1: Magnetic flux pattern in superlattice composed of 48 YBCO layers. The temperature of the sample at the record was $3.7 \mathrm{~K}$. A magnetic field of $4.3 \mathrm{mT}$ was applied after zero-field cooling. The polarizer and analyzer are uncrossed by 5 degrees to distinguish between positive (white) and negative (dark) magnetic fields.
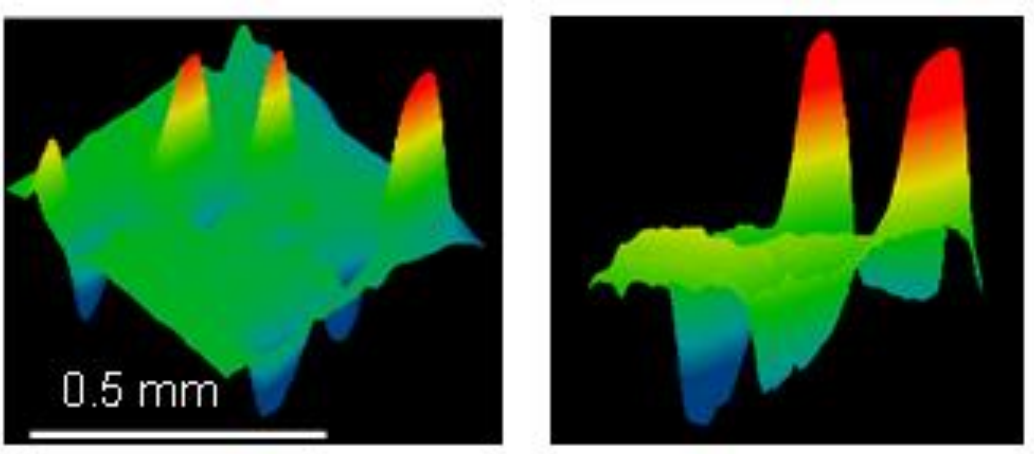

Figure 2: Color-coded 3D images of magnetic flux pattern in superlattice in the areas outlined by red rectangular frames in Fig. 1. Along the z-axis of the images, the intensity of the light is plotted. 


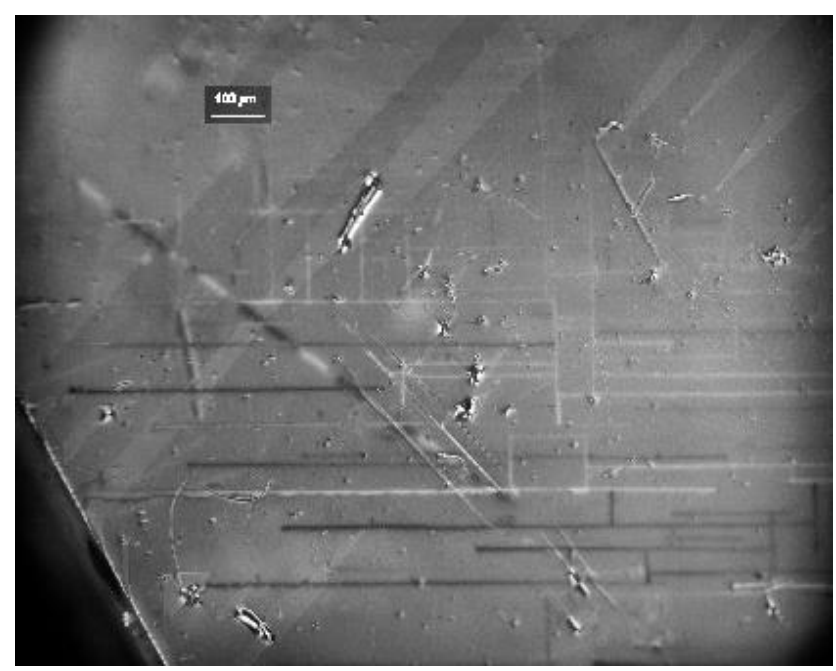

Figure 3: A stripy pattern (marked with red arrow) with periodical repetition of the fragments of positive and negative magnetic flux, on the background of weak horizontal and vertical lines, which belong to a network of nano-fractures in a superlattice with four layers of YBCO of a thickness of $750 \mathrm{~nm}$. The image was recorded at $68 \mathrm{~K}$ after application and removal of a very small magnetic field below $1 \mathrm{mT}$.

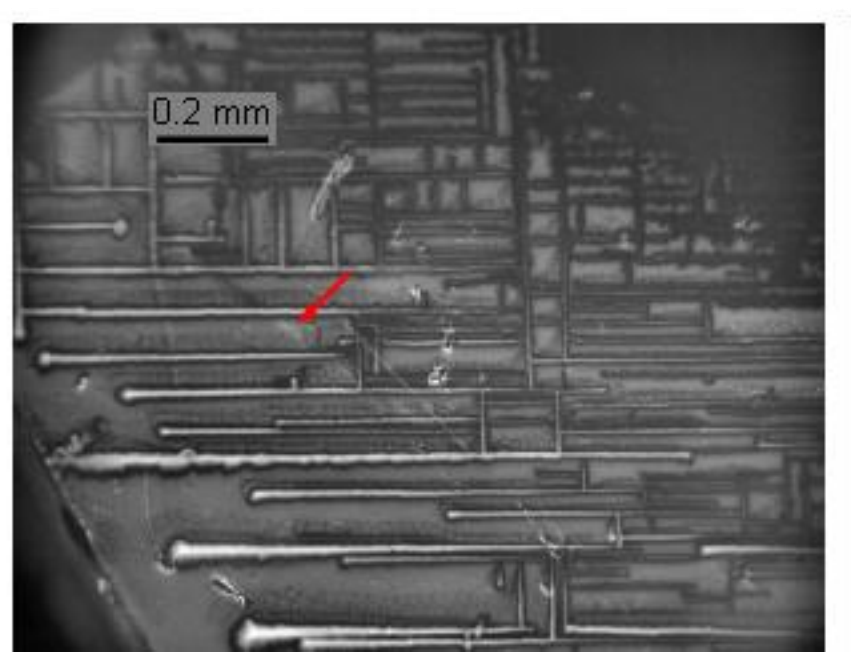

Figure 4: MOI image of the same sample as in Fig. 3. The image is recorded at $68 \mathrm{~K}$ with application of magnetic field of $6.8 \mathrm{mT}$. 


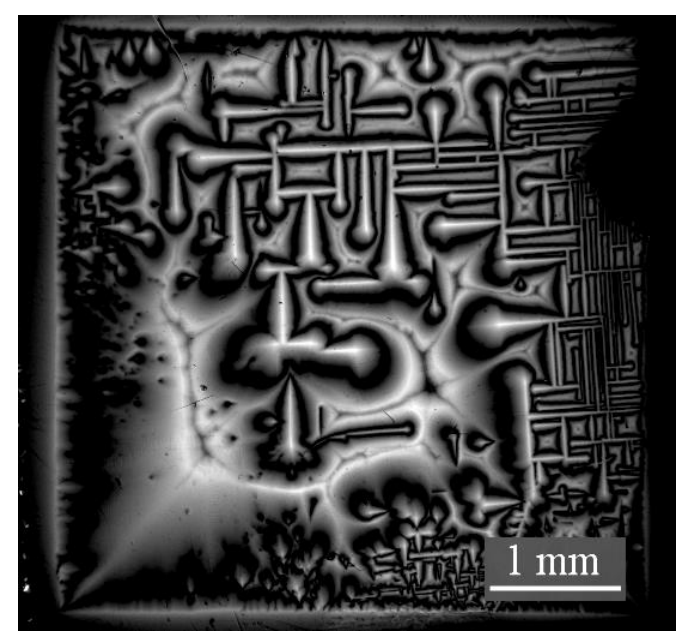

Figure 5: MOI image of the whole sample, top-left part of which is shown in Fig. 1. The image was recorded at $77.3 \mathrm{~K}$ after the application and removal of magnetic field of $85 \mathrm{mT}$.

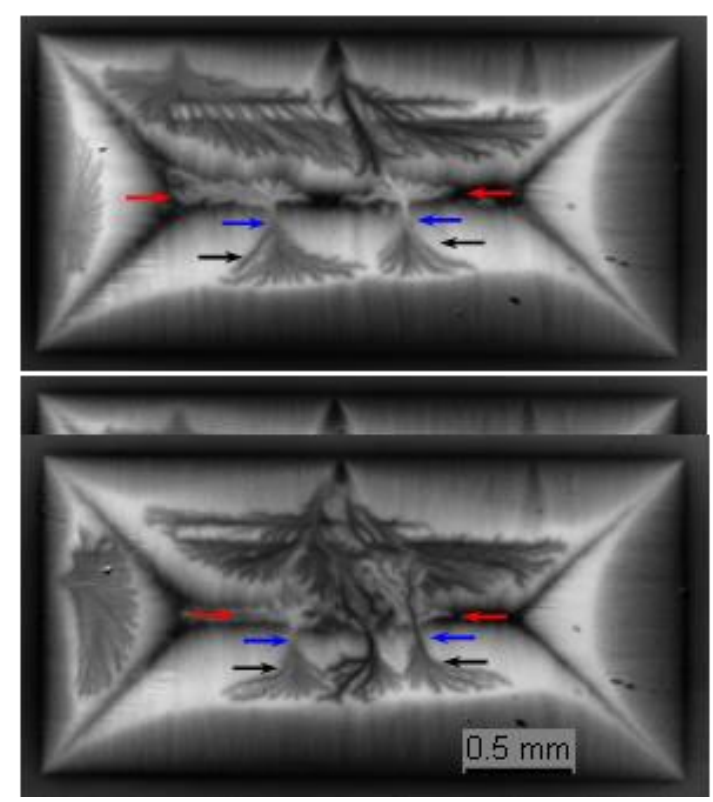

Figure 6: Dendritic flux avalanches with magnetic flux of opposite direction that are coming from the same points marked by blue arrows. Red arrows point to avalanches with positive and the black arrows point to avalanches with negative magnetic flux. 